\title{
Problems With Immunization Against Infectious Diseases
}

\author{
EDWARD A. MORTIMER, JR. \\ Case Western Reserve University School of Medicine, Cleveland, Ohio, USA
}

There is ample evidence that we are in difficulties with immunization programs, and that these difficulties jeopardize their current effectiveness and prospects for future development. Such evidence comprises less than optimum rates of immunization, the withdrawal of several manufacturers from vaccine production, extensive and increasing litigation involving physicians, pharmaceutical manufacturers, and public health agencies, disagreements about the informed consent process, wide public airing of legitimate scientific disagreements, and the like. Indeed, these difficulties have prompted this meeting. It is the purpose of this presentation to examine the nature and causes of some of these difficulties.

\section{TYPES OF VACCINES}

Vaccines, intended to protect against infection by inciting the recipient's own immunity as though the recipient had experienced the disease, are of five types.

The first type of vaccine comprises whole, killed microorganisms responsible for the disease in question. Bacterial vaccines of this type are pertussis, cholera, and typhoid. Viral vaccines of this type currently licensed are the influenza and the inactivated poliomyelitis vaccine (Salk).

Some bacterial vaccines (the second type) consist of fractions of the whole killed organisms; the recently licensed vaccines against pneumococcal pneumonia and meningococcal meningitis are of this type.

A third variety is the toxoid, of which there are two (diphtheria and tetanus). Toxoids comprise large amount of the inactivated toxin ("poison") elaborated by the offending organism. Toxoids induce immunity to the toxin without producing the serious symptoms of the disease, such as muscle spasms in tetanus (lockjaw).

A fourth variety is the live, attenuated organism; the measles, rubella (German measles), mumps, and oral poliomyelitis (Sabin) vaccines are of this type. Each of these produces infection with few or none of the manifestations of the disease. They are attenuated (weakened) by laboratory procedures.

The fifth type is the cross-reactive organism, a live microbe related immunologically to the disease organism and which, when given to man, produces a milder infection that protects against a serious one. Smallpox vaccine, derived from cowpox, and BCG for tuberculosis, derived from the bovine tuberculosis organism, are of this fifth type.

\section{VACCINES ARE NOT PERFECT}

Vaccines are no different from any drug in the sense that potential or actual risk of untoward effects is the rule. Moreover, $100 \%$ effectiveness cannot be guaranteed. Thus, for every vaccine some estimate of the benefit-risk ratio must be made. Unfortunately, as has been learned by the panels on bacterial and viral vaccines of the Bureau of Biologics of the FDA, data that permit such estimates with confidence in their precision are usually not available.
Table 1 shows the current US recommendations for the routine -immunization of children. It might be useful to examine each of these commonly used agents from the standpoint of what is and is not known about their efficacy and safety (the benefit-risk ratio). In terms of DTP, the combination of diphtheria and tetanus toxoids with pertussis vaccine, the pertussis component is currently the most controversial in terms of its benefits and risks. There is little question that tetanus toxoid is highly efficacious, inasmuch as when given in an appropriate dosage regimen it appears to be as close as $100 \%$ protective as any vaccine can be. Moreover, tetanus toxoid is quite safe, although review of the literature indicates that extraordinarily rare, fatal allergic reactions have been described. But the benefit-risk ratio of tetanus toxoid is very high; as high as one could ever hope to achieve when one administers a foreign protein to a human subject. Diphtheria toxoid, curiously, differs from tetanus toxoid in that it produces unpleasant reactions (such as fever) at what is probably a higher rate than does tetanus toxoid, but death and permanent disability have not been described. However, diphtheria toxoid is not as efficacious as tetanus toxoid; there are many reported instances of diphtheria in fully or partially immunized individuals, though such cases are milder and less apt to be fatal. An interesting problem that we face with diphtheria toxoid at the present time is a consequence of legislation introduced by the late Senator $\mathrm{Ke}$ fauver, which requires that all drugs, including biologicals, old or new, must be reviewed before licensure, not only for safety, but also for proven efficacy. Many of the studies of diphtheria toxoid, which first appeared in this country 55 years ago, do not meet current standards of study design and analysis, and, accordingly, it is impossible to establish the precise efficacy of diphtheria toxoid. Circumstantial evidence strongly suggests but does not prove that current low rates of diphtheria in the US and other countries in which the toxoid is widely used are a consequence to a large extent of immunization. In the light of this strong circumstantial evidence, it is difficult to justify the inconvenience and expense, to say nothing of the possible risks, of large field trials of diphtheria toxoid.

Pertussis vaccine is an entirely different story. This vaccine is crude and impure by the standards of many other immunizing agents. It comprises whole killed organisms, which contain many proteins of varying nature and possible toxicity. There is no question that pertussis vaccine is more reactive than we would like, and that some of these reactions are associated with permanent sequelae, such as disabling cerebral damage. Other reactions to pertussis vaccine, such as a peculiar high-pitched screaming that persists and occurs in some infants who receive the vaccine or excessive and worrisome sleepiness that occurs after the vaccine, are of unknown importance in terms of whether they induce permanent sequelae. Unfortunately, although these reactions attributable to pertussis vaccine have been known for some years, the precise rates at which they occur have not been defined. Estimates of the benefit-risk ratio of pertussis vaccine, in the light of these low but undesirable rates of reactivity, are further complicated by the fact that the protective efficacy of pertussis vaccine 
Table 1. Recommended schedule for the active immunization of normal infants and children

\begin{tabular}{ll}
\hline \multicolumn{1}{c}{ Age } & \multicolumn{1}{c}{ Vaccines } \\
\hline 2 months & DTP' and TOPV \\
4 months & DTP and TOPV \\
6 months & DTP (TOPV-optional) \\
15 months & Measles, rubella, and mumps \\
$11 / 2 \mathrm{yr}$ & DTP and TOPV \\
$4-6 \mathrm{yr}$ & DTP and TOPV \\
$14-16 \mathrm{yr}$ (and every $10 \mathrm{yr}$ thereaf- & Td $^{3}$ \\
ter) &
\end{tabular}

\footnotetext{
' Diphtheria and tetanus toxoids and pertussis vaccine, combined.

${ }^{2}$ Trivalent oral poliomyelitis vaccine. Contains all three immunologically distinct strains of attenuated poliomyelitis virus.

${ }^{3}$ Adult form of tetanus and diphtheria toxoids. The diphtheria toxoid component is reduced because of possible reactivity in individuals with several previous inoculations.
}

is open to challenge. As with many other important infectious diseases, mortality from pertussis declined in the US and elsewhere long before the vaccine appeared. In the US, the decline in pertussis mortality accelerated to the point that the number of deaths from pertussis in the US at the present time is negligible and, indeed, the decline subsequent to development of the vaccine was clearly and significantly from the statistical standpoint more rapid than that before the vaccine. But the unquestioned toxicity

$>$ of the vaccine, with permanent damage to a few recipients, and doubts about its efficacy have created controversy, particularly in Britain, about whether its routine use is currently justified. Here, again, we face a problem similar to that noted in relation to diphtheria toxoid; very few of the early studies of pertussis vaccine that appeared to provide evidence of efficacy meet current standards of study design.

As a consequence of the oral poliomyelitis vaccine the disease is close to eradication in the US. Thirty years ago, approximately 40,000 cases of poliomyelitis occurred annually in the US and about half of these resulted in paralysis. During the 3 years, 19521954, approximately 1700 individuals in the greater Cleveland area were admitted to hospitals with poliomyelitis; more than 100 succumbed to the disease and approximately 250 were left with residual respiratory impairment sufficient to require respiratory assistance indefinitely (the iron lung). In contrast, during the calendar year 1977, 20 cases of poliomyelitis occurred in the US, and so far in 1978, one case has been reported. Unfortunately, however, it appears that 14 of the 20 cases that occurred in 1977 were vaccine-induced. As a consequence of this, the question has been raised as to whether we should return to the injected inactivated poliomyelitis vaccine which, because the virus is killed, does not produce poliomyelitis. Inactivated vaccine has been used in Scandinavian countries; vaccine failures have not been observed. But Scandinavian populations exhibit rates of acceptance of the vaccine in excess of $95 \%$, in contrast to the United States where probably not many more than $75 \%$ of the population has received the full course of immunization with the oral poliomyelitis vaccine. With lower rates of immunization in the US, there is serious question in the minds of many whether the record achieved by inactivated vaccine in Scandinavia can be extrapolated to this country. Indeed, a small microepidemic of poliomyelitis in Sweden and a larger outbreak in the Netherlands involving 108 individuals, both occurring in groups who refused to accept inactivated poliomyelitis vaccine, suggests that inactivated vaccine provides considerably less community protection than the oral attenuated variety. The appearance of 108 cases of poliomyelitis in the Netherlands with a population of less than 14 million is equivalent to approximately 1650 cases in the US.

The live attenuated measles, mumps, and rubella vaccines, administered in combination (MMR), are also not perfect. In terms of the measles component of this triple vaccine, miniscule amounts of transplacental antibody interfere with getting a "take." For this reason, it was recently recommended that immunization be moved from 12 months to 15 months of age, to allow for greater decay in such maternal antibodies. Moreover, the measles vaccine is quite susceptible to loss of potency when exposed to light and if, during the chain of events from manufacturer to the patient, any breakdown in refrigeration occurs. Finally, in terms of risk, a rare but distressing and fatal complication of true measles, subacute sclerosing panencephalitis follows measles vaccine, though at a lower rate than that from the natural disease. An additional major problem is the fact that a number of teenagers in this country who neither received the vaccine nor experienced the natural disease are now susceptible to measles at an age when the disease is more severe. The possibility of fetal damage from the wild disease exists, but public health officers are reluctant to administer the vaccine because of possible fetal damage or confusion as to the cause of any abnormality in the infant.

Problems with the rubella (German measles) component of MMR are similar. The efficacy of the vaccine has recently been challenged and a failure rate much higher than one would like has been suggested. Concerns about the possibility of fetal abnormalities as a consequence of administering the vaccine during pregnancy have impeded efforts to immunize women of childbearing age. Moreover, transient arthritis, resembling rheumatoid arthritis, occurs not infrequently after the administration of rubella vaccine (as it does with the natural disease). In a few individuals, the arthritis has persisted and confusion, particularly from the liability standpoint, exists as to whether such cases of persistent arthritis represent sequelae of the rubella vaccine or the chance occurrence of true rheumatoid arthritis in association with vaccine administration, the more likely explanation.

Mumps vaccine, the third part of MMR, appears to be innocuous. But, again, questions have been raised about its failure to take.

Without going into detail, other vaccines are not perfect. Currently available influenza vaccines offer about $80 \%$ protection, and they are not without risk, as evidenced by the appearance of the Guillain-Barre syndrome in a few individuals after immunization with the swine flu vaccine. Other vaccines are even less efficacious; if we had to rely on typhoid vaccine, rather than sanitary engineering, for the control of typhoid fever, Georgetown University would currently have a large typhoid fever hospital. The best cholera vaccines are only $50 \%$ effective; for this reason, they are only used as an adjunct to sanitary measures in treatment of the disease. BCG vaccine for the control of tuberculosis is enormously difficult to evaluate; studies in the past have shown varied results. The most recent study, conducted under the auspices of the World Health Organization, has shown no effect whatsoever.

\section{PUBLIC EXPECTATIONS AND REALITY}

The remarkable achievements in the control of infectious disease, in which vaccines have had a major role, have created great expectations in the mind of the public. The ever-present specter of poliomyelitis each summe $e_{1}$ in the 30's and 40's has disappeared. The last case of smailpox in the US was 30 years ago. Parents no longer worry about diphtheria. Thus, the public has come to expect miracles from vaccines and from other infectious disease control measures. The miracle of the control of poliomyelitis-a truly remarkable achievement-has been clouded by the fact that the vaccine is not without some risks and by the fact that other vaccines and other means of control of infectious disease are not that efficacious and may not be without risk. In addition, the enormous publicity associated with the control of poliomyelitis by vaccines has augmented public expectations. Complicating this is the fact that we, in the medical profession, have been too willing to accept accolades and have failed to acknowledge that everything we do comprises a calculated risk. Thus, part of our trouble arises from public realization that we and our instruments are not perfect. 


\section{E INDIVIDUAL VERSUS THE PUBLIC}

In the past decade, it appears to me that we have seen increasing emphasis on the rights of the individual and deemphasis of the rights of the public. In terms of immunization, this has manifested itself by increased emphasis on the individual (or the individual's guardians) having responsibility for deciding whether a given immunization will or will not be administered. Known as informed consent, this process has caused considerable concern on the part of many individuals responsible for the operation of immunization programs. Is informed consent really possible? Do most patients or parents have the background to comprehend the pros and cons of a given procedure? Indeed, in some population groups in the US, it can be argued presently that there is greater risk from pertussis vaccine than from the disease itself. But the reason for this is that essentially everybody else in that population segment is immunized, and the individual child is protected from acquiring pertussis by this fact. If, in such a population, everyone individually refused pertussis immunization, the risk would increase enormously. Moreover, what do we do when the rights of the individual impinge upon the rights of the public? The economic consequences of many diseases preventable by immunization fall upon the public at large. For example, is the public really responsible when, for religious or other special reasons, a family refuses measles vaccine for their children and one develops measles encephalitis, requiring institutionalization?

All of this is enormously complicated by the current concern of the law and lawyers with process and not with end results. In my mind, many of the current difficulties with immunization stem from the law's concern with what is ritualistically legal and not with what is right. The case in Texas of Reyes vs. Wyeth, which contributed to Wyeth's withdrawal from vaccine manufacture, is a case in point. This 8-month-old infant, resident of a Texas community across the Rio Grande River from Mexico where an epidemic of poliomyelitis was occurring and which epidemic had crossed the river into Texas, developed paralytic poliomyelitis subsequent to receiving poliomyelitis vaccine in a community public health effort to stop the outbreak. The virus causing the disease was recovered from the infant, and laboratory studies of this virus indicated that it displayed the characteristics of the wild poliomyelitis virus then present in the community, and not those of the vaccine virus. The jury nonetheless decided that Wyeth was culpable. On appeal, the higher courts were unwilling to consider the fact that the virus displayed the characteristics of the wild virus, rather than the vaccine virus, because that was a decision of fact made by the jury. Decisions of fact, in the legal process, are rarely, if ever, challenged by higher courts.

Thus, it seems to me that the law, which has enormous power over what we do and don't do, is, in part, responsible for many of our difficulties and, moreover, impedes, rather than assists in, their solution.

\section{CATCH 22}

Physicians concerned with developing high rates of protection against infectious disease through public immunization programs are hampered by some apparent paradoxes in regulations and responsibilities. By law, children must go to school. By law or regulation, children must be immunized against six or seven childhood diseases (Table 1) in order to go to school. These are decisions made by the public and its representatives.

But, assuming that the benefits of widespread immunization programs far outweigh the risks and costs compared to alternatives, the public, which has established these requirements, does not assume responsibility for untoward events that may occur in association with them. If the rare, but nonetheless expected untoward event or reaction occurs to a vaccine, under our present situation it is the provider of the vaccine (the physician or the health department) and/or the pharmaceutical manufacturer who is found to bear the responsibility and from whom recompense comes. Why does not the public, which requires that children go to school and requires that they be immunized in order to go to school, not bear this responsibility?

\section{OTHER PROBLEMS}

There are many other problems with immunization. The infrequency with many of the diseases against which we immunize, such as poliomyelitis and diphtheria, has resulted in apathy not only on the part of the public but also on the part of many health providers. Clearly, this explains in part why rates of immunization in the US are not as high as we would like.

Public appetite for headlines, to which the media respond all too readily, has resulted in misunderstanding of many of the problems associated with immunization. For example, during the $24 \mathrm{hr}$ after community immunization against the swine flu in Cleveland, one individual who received the vaccine died, presumably of a heart attack. This made the front page as a major headline in one of the two newspapers. But, unfortunately, the newspaper never stopped to think about the possibility that in the particular segment of the population immunized on that day, one or more deaths might have been expected in the natural course of events. Indeed, examination of age-specific mortality rates in the population that was immunized suggested that two-and-a-half deaths, rather than one, were to have been expected.

Among other problems is the fact that current restrictions, litigation, and the like impede research by manufacturers toward the development of new, improved vaccines. It is well known that many manufacturers have dropped out of the vaccine business, partly because it is relatively unprofitable and partly because of liability issues. In 1977, for example, the wholesale value of all vaccines manufactured in the US was one-third that of Valium alone.

The solutions to these problems are not easy; their solutions, however, are not the responsibility of the medical profession alone.

\section{DISCUSSION}

The question of risks and benefits of polysaccharide vaccines such as the recently licensed pneumococcal vaccines was raised. It was concluded that these vaccines are recommended for special high risk groups. For example, the use of meningococcal vaccines in military recruits where epidemic spread has been observed, has reduced disease spread. Similarily, the use of pneumococcal vaccines are recommended for high risk groups such as children with sickle cell anemia or elderly adults with underlying cardiovascular disease; groups known to have an increased susceptibility to pneumoccal disease. Although not licensed, $\mathrm{H}$ influenzae polysaccharide vaccines have been shown to be quite effective in stimulating protective antibody in older children and adults, but appear to be ineffective in younger infants below 15 months of age.

The question of reimmunization for common childhood diseases was posed for children at the high school level. Recently there have been outbreaks of measles in such children, many of whom had been previously immunized in infancy. Therefore, the question of inadequate protection was raised. Dr. Sabin felt that in the case of polio, revaccination was not necessary based on studies which demonstrated the persistence of adequate levels of protective antibody. Dr. Mortimer explained that although the American Academy of Pediatrics does recommend four doses of oral poliovirus vaccine, two in the first year, one at $1 \frac{1}{2}$ years, and one at school entry, the fourth is intended as a filler, i.e., a precautionary measure to cover any child who may have missed one of the previous immunizations.

In the case of measles, however, Dr. Sabin felt that reimmunization of the high school group might be considered because about $85 \%$ of all reported cases are now seen in this group and this is the place where most of the spread occurs. This would break the chain of transmission of measles virus. He also recommended a continued effort to increase the vaccination of preschool children.

In regard to revaccination of adults with polio virus vaccine, Dr. Sabin felt this was not necessary. 
A question was raised regarding the Surgeon General's report that the incidence of paralytic disease that results from oral polio vaccine is higher in individuals over 18 years of age. Dr. Sabin commented that in his opinion, the statement is totally biased, based on data taken from the initial immunization campaign, which even then could not be borne out. Dr. Sabin stated that he had analyzed many of the so-called vaccine-associated cases of paralytic disease and resolved that there was insufficient evidence to support this conclusion.

Copyright ( 1979 International Pediatric Research Foundation, Inc. $0031-3998 / 79 / 1305-684 \$ 02.00 / 0$
A comment was directed at the role of influenza virus in influenza associated diseases. Dr. Douglas felt that the low rates of influenza which are reported may be reflective of interpretations made on virus isolation. Much higher rates, approximately $50-85 \%$, can be made if one bases interpretation on seroconversion. Dr. Sabin agreed with this point but nevertheless felt that in typical influenza outbreaks, upwards of $50 \%$ or more of those considered typical had no indication of infection by influenza virus either by viral isolation or seroconversion. 